Discrete Comput Geom 28:411-425 (2002)

DOI: $10.1007 / \mathrm{s} 00454-002-2819-\mathrm{z}$

Geometry

\title{
The Kernel of the Adjacency Matrix of a Rectangular Mesh*
}

\author{
Carlos Tomei and Tania Vieira \\ Departamento de Matemática, \\ Pontifícia Universidade Católica do Rio de Janeiro, \\ Rua Marqués de Sáo Vicente 226, Gávea, \\ Cap 22453, Rio de Janeiro, Brazil \\ \{tomei,tvieira\}@mat.puc-rio.br
}

\begin{abstract}
Given an $m \times n$ rectangular mesh, its adjacency matrix $A$, having only integer entries, may be interpreted as a map between vector spaces over an arbitrary field $K$. We describe the kernel of $A$ : it is a direct sum of two natural subspaces whose dimensions are equal to $\lceil c / 2\rceil$ and $\lfloor c / 2\rfloor$, where $c=\operatorname{gcd}(m+1, n+1)-1$. We show that there are bases to both vector spaces, with entries equal to 0,1 and -1 . When $K=\mathbb{Z} /(2)$, the kernel elements of these subspaces are described by rectangular tilings of a special kind. As a corollary, we count the number of tilings of a rectangle of integer sides with a specified set of tiles.
\end{abstract}

\section{Introduction}

A basic combinatorial problem associated to a quadriculated surface is the counting of its tilings by dominos. Tilings of rectangles of integral sides, for example, were counted by Kasteleyn $[\mathrm{K}]$ in his solution of the Ising model. More generally, Kasteleyn showed how to alter slightly the adjacency matrix of a quadriculated disk so that the determinant of the resulting Kasteleyn matrix equals the desired count. Here, the adjacency matrix specifies which squares of the quadriculated surface share an edge. Tilings of other surfaces have been counted: the Aztec diamond is another example, presented in [EKLP]. Moreover, tilings have been $q$-counted, according to a height variable $q$ ([EKLP] and [STCR]).

In [DT] the authors proved, in a different language, that the (-1)-counting of tilings of a quadriculated disk is always equal to 0,1 , or -1 . Their interest in the issue stemmed from the fact that the adjacency matrix $A$ of a quadriculated surface is closely related to a standard discretization of the Laplacian with Dirichlet boundary conditions [C].

\footnotetext{
* The first author acknowledges support from CNPq and FAPERJ, the second acknowledges support from CNPq.
} 
The result indicates a spectral rigidity for the discrete Laplacian, which so far has no interpretation in the continuous case. Further study of this rigidity led to the consideration of additional properties of (-1)-counting, both on disks [STCR] and on annuli [ST1].

Since the adjacency matrix $A$ has only integer entries, it may be interpreted as a linear transformation $A_{K}$ between vector spaces over an arbitrary field $K$. By coloring squares of the quadriculated disk black and white in a chessboard fashion, it is easy to see that $A_{K}$ takes vectors supported on the set of black squares (forming a subspace $B_{K}$ ) to vectors supported on white squares (in $W_{K}$ ), and vice versa. Thus, $A_{K}$ splits naturally into two linear maps, $B W_{K}: B_{K} \rightarrow W_{K}$ and $W B_{K}: W_{K} \rightarrow B_{K}$ and the kernel of $A_{K}$ is the direct sum of the kernels of $B W_{K}$ and $W B_{K}$.

In this paper we describe the kernel of $A_{K}$ in the special case of an $m \times n$ rectangle quadriculated by $(m n)$ squares of side of length 1 . Suppose that the left lower square is painted black. As usual, the standard roundoff operations are denoted by $\lceil x\rceil$ and $\lfloor x\rfloor$.

Theorem 1. For an $m \times n$ rectangle quadriculated by unit squares, $\operatorname{dim} \operatorname{ker} B W_{K}=$ $\lceil c / 2\rceil$ and $\operatorname{dim} \operatorname{ker} W B_{K}=\lfloor c / 2\rfloor$, where $c=\operatorname{gcd}(m+1, n+1)-1$. There is a basis to each kernel for which all vector coordinates are equal to 0,1 or -1 .

More recently [ST2], it was shown that, for an arbitrary quadriculated disk, both matrices $B W_{K}$ and $W B_{K}$ admit $L D U$-type decompositions, where the matrices $L, D$ and $U$ have all entries equal to 0,1 or -1 . From this result, the fact that $\operatorname{dim} \operatorname{ker} B W_{K}$ and $\operatorname{dim} \operatorname{ker} W B_{K}$ are independent of $K$ and the existence of bases for the kernels with entries equal to 0,1 and -1 follows. The decomposition also implies the rigidity result in [DT].

When $K=\mathbb{Z} /(2)$, the discretization of the Laplacian essentially coincides with the adjacency matrix $A_{\mathbb{Z} /(2)}$ and we call the elements on $\operatorname{ker} B W$ and $\operatorname{ker} W B$ the polarized $\mathbb{Z} /(2)$-harmonic functions, supported respectively on black and white points. Such objects have already been considered in [DT] as a technical tool. Draw the $m \times n$ rectangle so that the integer points of the auxiliary rectangle $R=[0, m-1] \times[0, n-1] \subset \mathbb{R}^{2}$ correspond to the centers of the squares. Consider tilings of $R$ by tiles given in Fig. 1 . Tiles can be rotated by integer multiples of $\pi / 2$ and their dashed sides ought to belong to $\partial R$, the boundary of $R$. The length of sides is indicated in the picture, angles must be $\pi / 4, \pi / 2$ or $3 \pi / 4$. Figure $2(1)$ shows a tiling of the $7 \times 4$ rectangle.

Integer points of $R$ inherit the coloration of the squares (in particular, the origin is black). As we shall see in Proposition 2, tilings of $R$ are such that the vertices of all tiles
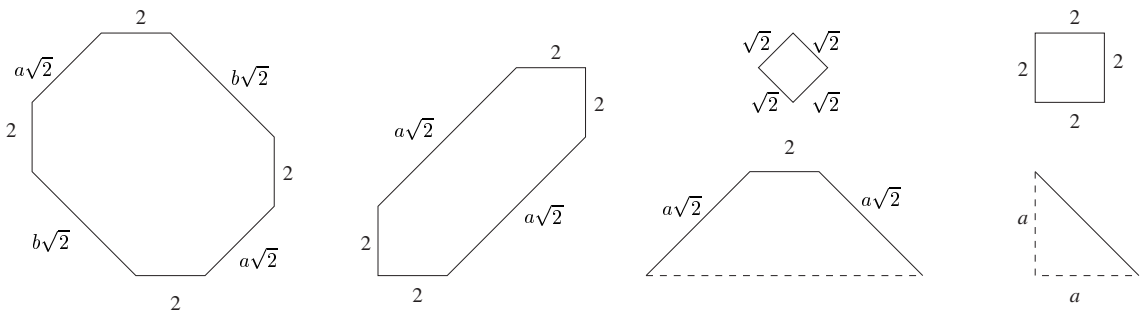

Fig. 1. The tiles; $a$ and $b$ are integers. 


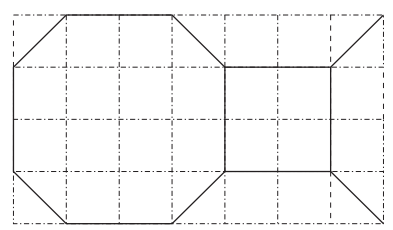

(1)

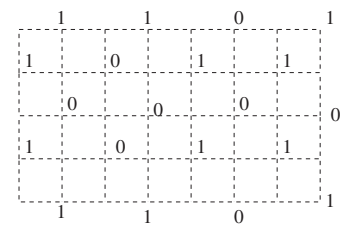

(2)

Fig. 2. A tiling and a polarized $\mathbb{Z} /(2)$-harmonic function of the $7 \times 4$ rectangle.

have integer coordinates and, with the possible exception of the corners of $R$, all vertices have the same color. Thus, the set of all tilings $\mathcal{T}$ naturally splits into two sets, $\mathcal{T}_{b}$ and $\mathcal{T}_{w}$. Polarized $\mathbb{Z} /(2)$-harmonic functions are visually very simple: this is the content of the theorem below.

\section{Theorem 2. There are bijections}

$$
\Phi_{b}: \operatorname{ker} B W_{\mathbb{Z} /(2)} \backslash\{0\} \rightarrow \mathcal{T}_{b} \quad \text { and } \quad \Phi_{w}: \operatorname{ker} W B_{\mathbb{Z} /(2)} \backslash\{0\} \rightarrow \mathcal{T}_{w}
$$

Combining both theorems, we obtain the number of elements in $\mathcal{T}_{b}$ and $\mathcal{T}_{w}$. Also, given a tiling (i.e., a polarized $\mathbb{Z} /(2)$-harmonic function), there is a lifting to an element of $\operatorname{ker} B W_{K} \cup \operatorname{ker} W B_{K}$, having only 0,1 and -1 as entries. This correspondence is essentially bijective: lifting of a vector can take at most two values $v$ and $-v$. This is the content of Theorem 4. Lifting first appeared in [DT].

\section{Kernel Vectors}

As before, let $R=[0, m-1] \times[0, n-1] \subset \mathbb{R}^{2}$. Adjacency in the $m \times n$ rectangular graph $G=R \cap(\mathbb{Z} \times \mathbb{Z})$ is the natural one: $(x, y)$ and $\left(x^{\prime}, y^{\prime}\right)$ are adjacent if and only if $x=x^{\prime}, y=y^{\prime} \pm 1$ or $x=x^{\prime} \pm 1, y=y^{\prime}$. We call points the elements of $G$ : vertices will be the corners of tiles. Color a point $(x, y) \in G$ black (resp. white) if $x+y$ is even (resp. odd): thus, the origin is black. Number black and white points in $G$ from 1 to $n_{b}$ and from 1 to $n_{w}$. Set $B W$ to be the $n_{w} \times n_{b}$ black-to-white adjacency matrix: $B W_{w b}=1$ if point $b$ is adjacent to point $w$; otherwise, $B W_{w b}=0$. Similarly, define $W B$, the $n_{b} \times n_{w}$ white-to-black adjacency matrix of $G$. Since both matrices $B W$ and $W B$ only have integer entries, they may be interpreted as linear transformations between vector spaces over an arbitrary field $K$.

We compute the dimensions of the kernels of $B W$ and $W B$ and show they are independent of the base field $K$. The kernel elements admit two special symmetries, which we now describe. Given a vector $v$ belonging to $K^{m n}$, denote by $\sigma_{i, j}$ the sum of the coordinates of $v$ at the four neighbors of $(i, j)$ in $G, \sigma_{i, j}=v_{i, j-1}+v_{i, j+1}+v_{i-1, j}+v_{i+1, j}$. Here $v_{i, j}=0$ if $(i, j) \notin G$. Recall that the kernel of the adjacency matrix $A$ of $G$ is the direct sum of the kernels of $B W$ and $W B$. Clearly, $v \in \operatorname{ker} A$ if and only if $\sigma(i, j)=0$ for all points $(i, j) \in G$. Without loss, the sides of the rectangular graph satisfy $m \geq n$. 
Proposition 1. Let $v \in \operatorname{ker} A$. Then:

(i) $v_{i, j}=v_{j, i}$ (resp. $\left.-v_{j, i}\right)$ if both points belong to $G$ and $i+j$ is even (resp. odd).

(ii) If $v$ equals zero along the column $x=m_{0}$, then $v_{m_{0}-i, j}=-v_{m_{0}+i, j}$, if both points belong to $G$.

There are analogous symmetries across the other diagonals of the graph, and across rows of zeros of $v$.

Proof. Compare values of $v$ along the lines $y=x+1$ and $y=x-1$. Since $\sigma_{0,0}=0$, we must have $v_{1,0}=-v_{0,1}$. Sequentially, $\sigma_{1,1}=0, \sigma_{2,2}=0, \ldots$ imply in turn $v_{2,1}=-v_{1,2}$, $v_{3,2}=-v_{2,3}, \ldots$. Compare now values of $v$ along the lines $y=x+3$ and $y=x-3$ : making use of $\sigma_{2,0}=0=\sigma_{0,2}$, we have $v_{0,3}=-v_{3,0}, v_{1,4}=-v_{4,1}, \ldots$ Proceed until the lines $y=x \pm(2 k+1)$ cover the square $[0, n-1] \times[0, n-1]$ : this proves the statement for coordinates $(i, j)$ for which $i+j$ is odd. The statement for coordinates with $i+j$ even follows from a similar sequential computation along lines $y=x \pm 2 k$ : to start off the induction argument, notice that $v_{i, j}=v_{j, i}$ along the diagonal $y=x$.

The proof of (ii) is a simple induction on $i$. Case $i=1$ is as hard as the general case, and follows from expanding $0=\sigma_{m_{0}, j}=v_{m_{0}-1, j}+v_{m_{0}+1, j}$.

Consider the $m \times n$ rectangular graph $G$ and let $c=\operatorname{gcd}(m+1, n+1)-1$. The grid $\mathcal{G}$ of $G$ is the set of points in $G$ belonging to the union of the horizontal lines $y=k(c+1)-1$ with the vertical lines $x=k(c+1)-1$, for integer $k$. The graph $G$, after removal of its grid $\mathcal{G}$, splits into $c \times c$ squares, called fundamental squares. Figure 3 shows the grid and the fundamental squares for the $14 \times 9$ rectangular graph. The figure also contains the description of an element $v$ in ker $W B$, interpreted as a vector with coordinates over the field $\mathbb{Z} /(2)$ of two elements—squares indicate the coordinates with nonzero entries. The edges connecting points in $G$ are drawn to provide a visual indication of the symmetries of this kernel element. As we shall prove later, $\mathbb{Z} /(2)$-polarized harmonic functions correspond to tilings of $R$ : Fig. 3 is indeed the tiling associated to $v$.

As before, fix an arbitrary base field $K$.

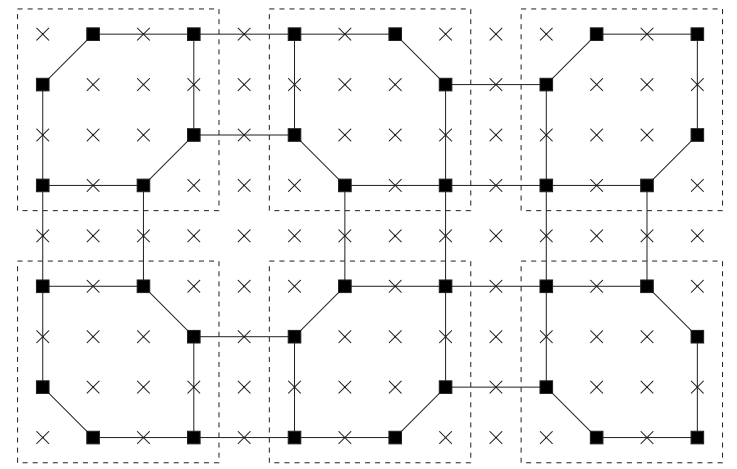

Fig. 3. Grid, fundamental squares and symmetries. 
Theorem 3. Let $G$ be the $m \times n$ rectangular graph with grid $\mathcal{G}$ and adjacency matrices $B W_{m, n}$ and $W B_{m, n}$. Let $v \in \operatorname{ker} B W_{m, n}\left(r e s p . \operatorname{ker} W B_{m, n}\right)$. Then:

(i) The vector $v$ is identically zero on $\mathcal{G}$.

(ii) The restriction of $v$ to each fundamental square lies in the kernel of the matrix $B W_{c, c}\left(\right.$ resp. $\left.W B_{c, c}\right)$ of this square.

(iii) Let $s_{1}$ and $s_{2}$ be two adjacent fundamental squares, bounded by a common row or column $\ell$ of $\mathcal{G}$. Then the restriction of $v$ to $s_{2}$ is the odd extension of the restriction of $v$ to $s_{1}$ across $\ell$.

(iv) Let $G_{c, c}$ be the $c \times c$ square graph. Then

$$
2 \operatorname{dim} \operatorname{ker} B W_{c, c}=c+(c \bmod 2) \text { and } 2 \operatorname{dim} \operatorname{ker} W B_{c, c}=c-(c \bmod 2) .
$$

(v) $\operatorname{dim} \operatorname{ker} B W_{m, n}=\operatorname{dim} \operatorname{ker} B W_{c, c}$ and $\operatorname{dim} \operatorname{ker} W B_{m, n}=\operatorname{dim} \operatorname{ker} W B_{c, c}$.

(vi) There are bases for $\operatorname{ker} B W_{m, n}$ and $W B_{m, n}$ consisting of vectors having coordinates taking only the values 0,1 and -1 .

In particular, the dimensions of $\operatorname{ker} B W_{m, n}$ and $\operatorname{ker} W B_{m, n}$ are independent of the base field $K$.

Proof. We shall prove (i)-(iii) by induction on the set of pairs $(m, n)$ for which $\operatorname{gcd}(m+1, n+1)=c+1$ is fixed, ordered lexicographically. For the starting point $m=n=c$, the result is obvious. Suppose that $v^{m, n} \in \operatorname{ker} B W_{m, n}$, in a self-explanatory notation. From the symmetry (i) of the previous proposition, the restriction of $v^{m, n}$ to column $x=n$ is zero (recall that $m \geq n$ ). Indeed, for $v=v^{m, n}$, since

$$
\begin{aligned}
& 0=\sigma_{n-1, j}=v_{n-2, j}+v_{n-1, j-1}+v_{n-1, j+1}, \\
& 0=\sigma_{j, n-1}=v_{j, n-2}+v_{j-1, n-1}+v_{j+1, n-1}+v_{j, n},
\end{aligned}
$$

we must have $v_{j, n}=0$. Removal of column $x=n$ from $G=G_{m, n}$ gives rise to two connected subgraphs: a square $G_{n, n}$ containing the first $n$ columns of $G$ and a possibly rectangular subgraph $G_{m-n-1, n}$ containing only the last $m-n-1$ columns. Also, by making use of the column of zeros and the fact that $v^{m, n} \in \operatorname{ker} B W_{m, n}$, we have that the restriction $v^{m-n-1, n}$ of $v^{m, n}$ to $G_{m-n-1, n}$ belongs to $\operatorname{ker} B W_{m-n-1, n}$. Now, $\operatorname{gcd}(m-n-1+1, n+1)=c+1$ : by induction, then $v^{m-n-1, n}$ has zeros on the grid of $G_{m-n-1, n}$. Thus, the nonzero entries of $v^{m-n-1, n}$ belong to $c \times c$ squares framed by points in which $v^{m-n-1, n}$ is zero. Now using symmetry (ii), mirroring these squares across columns $n, n-c-1 \ldots$, we see that the square $G_{n, n}$ also splits in $c \times c$ squares with the same property. Conversely, taking odd extensions of a kernel element on the fundamental square across the grid obtains a kernel element on the full rectangle: this proves (v). A vector in $\operatorname{ker} B W_{c, c}$ (or in $\operatorname{ker} W B_{c, c}$ ) is clearly determined by its values along the first column $x=0$ of $G_{c, c}$. The fact that any choice of values along this column indeed gives rise to a kernel vector follows from the diagonal symmetry (i) of Proposition 1-this proves (iv). A special choice of vectors along the first column of $G_{c, c}$ obtains the basis of kernel vectors with coordinates 0,1 and -1 . 


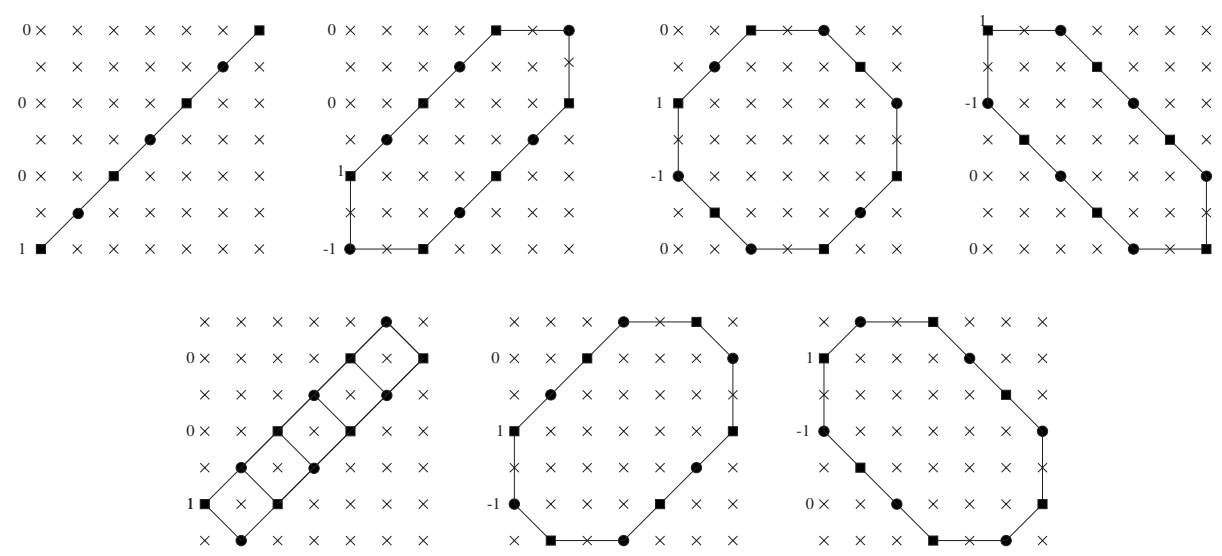

Fig. 4. Bases for $\operatorname{ker} B W$ and for ker $W B$.

For ker $B W_{c, c}$, they are $e_{1}, e_{3}-e_{1}, e_{5}-e_{3}, \ldots, e_{c+(c \bmod 2)-1}-e_{c+(c \bmod 2)-3}$ and for ker $W B_{c, c}, e_{2}, e_{4}-e_{2}, \ldots, e_{c+(c \bmod 2)-2}-e_{c+(c \bmod 2)-4}$. The details are left to the reader: Fig. 4 describes the simple bases for $G_{7,7}$. Squares and circles correspond to 1's and -1's, respectively.

\section{Basic Properties of Tilings}

Tiles are the polygons (topologically, closed disks) listed in Fig. 1. A tiling of $R=$ $[0, m-1] \times[0, n-1]$ is a covering of $R$ by tiles which overlap at their boundaries. Points of $\mathbb{Z} \times \mathbb{Z}$ are called integral points.

Proposition 2. Vertices of tiles of a tiling $T$ are always integral. Also, with the possible exception of the four corners of $R$, all vertices of tiles in $T$ are of the same color.

Thus $\mathcal{T}=\mathcal{T}_{b} \cup \mathcal{T}_{w}$, where $\mathcal{T}$ is the set of tilings of $R$, and $\mathcal{T}_{b}$ and $\mathcal{T}_{w}$ are the sets of tilings whose tiles are black and white.

Proof. From the shape of the tiles, if one vertex sits on an integral point, all other vertices in a tile do. Suppose now that $T$ contains a tile with some nonintegral vertex. Let $N$ be the region of $R$ covered by tiles of $T$ having nonintegral vertices: $N$ is a polygonal region which does not contain the corners of $R$-corners belong to tiles with integral vertices. Let $v$ be a vertex of $N$ : then it must also be the vertex of some tile outside $N$-a contradiction. Thus, all vertices are integral.

Again from the shape of tiles, all vertices of a tile have the same color (unless the tile is a right triangle with small sides of odd length) — call this the color of the tile (for triangles, the color of the tile is the color of the vertices not sitting at the right angle). The right angle vertex of a triangle necessarily sits on a corner of $R$, and we do not have to consider it in what follows. 
Again, with the possible exception of sides of triangles, all other horizontal or vertical sides are of even length. Thus, all tiles with a side lying on $\partial R$ have the same color $c$. Let $C$ be the region covered by tiles of color $c$, and let $D$ be its complement in $R$. If $D$ is not empty, take $v$ to be one of its vertices: since $v \in D$ is not a corner of $R$, it must have the colors of tiles both in $C$ and $D$-again a contradiction.

\section{Defining the Bijections $\Phi_{b}$ and $\Phi_{w}$}

For now on, the base field $K$ is $\mathbb{Z} /(2)$. The rest of the paper is dedicated to the proof of Theorem 2.

Let $G$ be the rectangular graph associated to $R$ and take $u \in \operatorname{ker} B W \backslash\{0\}$. A black point $b \in G$ is an active point of $u$ if $u(b)=1$. Denote by $A_{u}$ the set of active points of $u$. In $A_{u}$ consider the following adjacency relation (Fig. 5). Two points are adjacent if both conditions are satisfied: (i) both points have a common white neighbor $w$ in $G$; (ii) both points form a right angle with $w$ or they are the only active neighbors of $w$.

A splitting of $R$ is a decomposition in chunks, i.e., polygons with integral vertices, which are not necessarily tiles. Given a vector $u \in \operatorname{ker} B W \backslash\{0\}$, let $\Phi_{b}(u)=$ $\operatorname{int}(R) \backslash\left\{\overline{b_{1} b_{2}} \mid b_{1}\right.$ and $b_{2}$ are adjacent in $\left.A_{u}\right\}$. Said differently, draw straight line segments joining adjacent points in $A_{u}$. In Section 4.1 we will see that this construction obtains a splitting $\Phi_{b}(u)$ of $R$. In Section 4.3 more will be proved: $\Phi_{b}(u)$ is actually a tiling, i.e., its chunks are tiles.

\subsection{Geometry of $A_{u}$}

Let $b \in A_{u}$ be a black point in the interior of $R$. The star centered at $b$ is the set of points in $A_{u}$ which are adjacent to $b$, together with the segments joining $b$ to these points. The lemma below follows by exhausting possibilities.

Lemma 1. Let $b \in A_{u}$ be an interior point of $G$. Up to rotations and reflections, the stars centered at $b$ are listed in Fig. 6.

We point out that all stars in the list indeed occur in tilings.

Lemma 2. Every point of $A_{u}$ which is not a corner of $R$ has (at least) two neighbors in $A_{u}$. Also, segments $\overline{b_{1} b_{2}}$ and $\overline{b_{3} b_{4}}$ in $A_{u}$ joining four distinct points do not intercept.

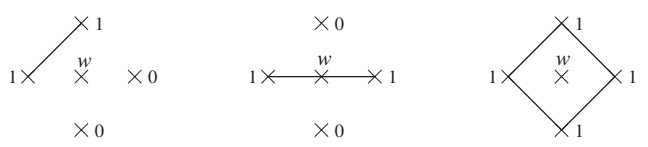

Fig. 5. Adjacencies in $A_{u}$. 




(1)

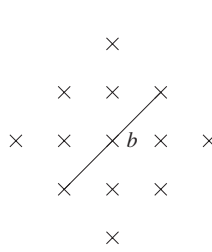

(4)



( 2 )

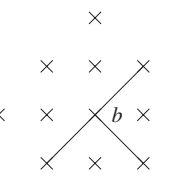

(5)

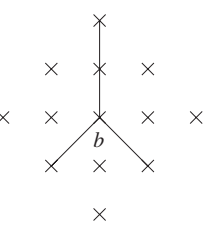

( 3 )

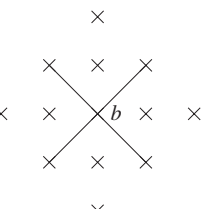

(6)

Fig. 6. Stars centered at $b$.

Proof. Let $b$ be a point in $A_{u}$ which is not a corner of $R$. Then $b$ has two neighbors in $G, w_{1}$ and $w_{2}$, so that the three points are collinear. Since $b$ is an active neighbor of $w_{1}$ and $w_{2}$ and $u \in \operatorname{ker} B W, w_{1}$ and $w_{2}$ have other active black neighbors in $G$. Let $b_{1}$ (resp. $b_{2}$ ) be the active neighbor of $w_{1}$ (resp. $w_{2}$ ) closest to $b$. Notice that $b_{1}$ and $b_{2}$ are distinct, since $b$ is the only black neighbor common to $w_{1}$ and $w_{2}$. Thus, $b$ is adjacent in $A_{u}$ to at least two points $b_{1}$ and $b_{2}$.

We now consider the second statement. From the definition of the adjacency in $A_{u}$, the possible segments joining active points are given in Fig. 7. Since the four points are distinct, segments may not meet at endpoints. Also, as all points have the same color, the only plausible intersection of segments are listed in Fig. 7. By direct inspection, each such intersection violates the definition of adjacency in $A_{u}$.

Proposition 3. The closure of the connected components of $\Phi_{b}(u)$ are convex polygons with vertices in $A_{u}$ or in the corners of $R$. In particular, $\Phi_{b}(u)$ is indeed a splitting.

Proof. From Lemma 2, the juxtaposition of segments in $A_{u}$, together with $\partial R$, consists of closed polygonal curves and the vertices of the connected components in the statement are either in $A_{u}$ or in the corners of $R$. From the classification of stars, convexity follows at interior vertices: for boundary vertices, convexity is trivial.

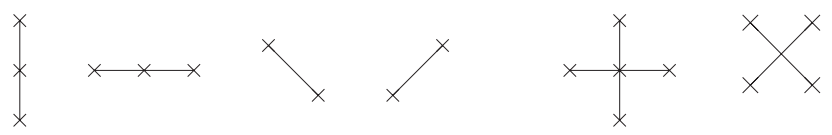

Fig. 7. Segments and their possible intersections. 


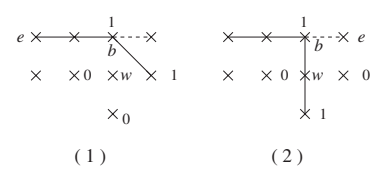

Fig. 8. Mixed segments do not exist; dashed segments are inactive.

\subsection{The Possible Sides and Angles of Chunks of $\Phi_{b}(u)$}

Sides of chunks of a splitting $\Phi_{b}(u)$ are active if they consist only of segments of $A_{u}$. Inactive sides are those which contain no segment of $A_{u}$. The remaining sides are called mixed. Inactive and mixed sides must lie in $\partial R$, since sides in $\Phi_{b}(u)$ are the union of segments in $A_{u}$ with segments in $\partial R$.

Lemma 3. There are no mixed sides.

Proof. Suppose $e$ is a mixed side of a chunk in $\Phi_{b}(u)$. Without loss, suppose $e$ belongs to the upper side of $R$. The $m \times n$ rectangular graph $G$ ought to include points immediately below $e$, since $m, n>1$. Let $b$ be a point of $e$ joining an active and an inactive segment, and let $w$ denote the white point below $b$. Figure 8 shows the possible active neighbors of $w$ : any possible choice implies the existence of a segment in $A_{u}$ meeting $e$ at $b$ : in particular, $e$ may not be a side of a chunk.

Lemma 4. Horizontal or vertical active sides of chunks in $\Phi_{b}(u)$ have length 2.

Proof. Consider a horizontal active side $h$ of a chunk of $\Phi_{b}(u)$. From the adjacency relation in $A_{u}, h$ has even length. Suppose $h$ has length greater than 2, and take $e$ in $h$ joining two active points at distance 4 .

As in the previous lemma, we may suppose that the integral points immediately under $h$ belong to $G$. The midpoint of $e$ has at least one white neighbor $w \in G$, as shown in Fig. 9. From the adjacency relation in $A_{u}$, the side neighbors of $w$ are inactive. There is only one possibility: the other active neighbor of $w$ must be $b$, as in Fig. 9. However, then the vertical segment in the same figure ought to be a segment in $A_{u}$, contradicting the fact that $e$ lies in a side of a chunk.

The angles of chunks are formed by the segments listed in Fig. 7 combined with segments in $\partial R$. Our next step will be the classification of angles according to activity of their sides. Angles defined by an active and an inactive side are mixed angles. From

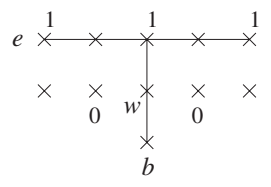

Fig. 9. Horizontal sides. 


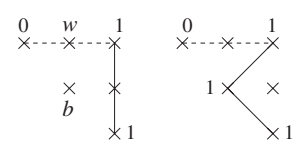

Fig. 10. An inexistent right angle.

the adjacency relation in $A_{u}$, angles of $\pi / 4$ in $\Phi_{b}(u)$ are mixed and angles of $3 \pi / 4$ are not. We are left with considering right angles.

Lemma 5. Right angles are not mixed.

Proof. Consider first the case in which the sides of the angle are horizontal and vertical, as in Fig. 10. The inactive side must then belong to $\partial R$. The number of active neighbors of $w$ must be even, and hence $u(b)=1$. However, then the adjacency relation in $A_{u}$ implies the presence of active segments as in Fig. 10.

The other kind of right angle has diagonal sides: these sides may not belong to $\partial R$ and thus have to be active. Indeed, by the adjacency relation in $A_{u}$, this kind of right angle may only occur in square chunks of sides with length $\sqrt{2}$.

Adding up, Fig. 11 lists all possible angles of chunks in $\Phi_{b}(u)$, taking into account the activity of each side.

Lemma 6. The number of $\pi / 4$ angles in a chunk of a splitting $\Phi_{b}(u)$ is even.

Proof. Lemma 3 excludes the possibility of mixed sides. Clearly, every chunk ought to have an even number of mixed angles, and the only mixed angles measure $\pi / 4$.

Lemma 7. Let $C$ be a chunk in $\Phi_{b}(u)$ with a side s common to two right angles which is active and either horizontal or vertical. Then $C$ is a square with sides of length 2.

Proof. Lemma 4 implies that the side $s$ has length 2. In Fig. 12(1), $b$ is not active, again in accordance with the adjacency relation in $A_{u}$. Thus, the only active neighbors of $w$ are adjacent in $A_{u}$.

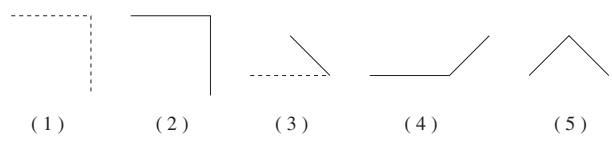

Fig. 11. Angles; active and inactive sides. 


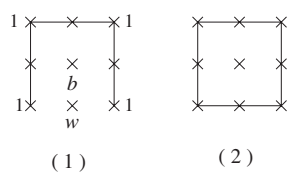

Fig. 12. (1) implies (2).

\subsection{Chunks in $\Phi_{b}(u)$ Are Tiles}

Since the smallest external angle of a chunk is $\pi / 4$ (Fig. 11) chunks of $\Phi_{b}(u)$ have at most eight sides-we now classify chunks.

Octagons. All inner angles have to measure $3 \pi / 4$. Horizontal and vertical sides have length 2 since they are active (Lemma 4) and thus octagons must be as in Fig. 1.

There are no heptagonal chunks. A heptagonal chunk should have six angles measuring $3 \pi / 4$ and one measuring $\pi / 2$ (Fig. 13). Horizontal and vertical sides must be active and have length 2 . From Fig. 13, adding coordinates on both axis, $a^{\prime}-b^{\prime}-c^{\prime}=0$ and $-a^{\prime}-b^{\prime}+c^{\prime}=0$. Thus, $a-b-c=0$ and $-a-b+c=0$, and hence $b=0$ : one side is gone.

Hexagons. From Lemma 6, chunks of $\Phi_{b}(u)$ have an even number of angles measuring $\pi / 4$. Thus, hexagons must have four $3 \pi / 4$ angles and two $\pi / 2$ angles. Angles ought to be ordered as in Fig. 1: from Lemma 7, the right angles have to be separated.

There are no pentagonal chunks. Again, from Lemma 6, a pentagon should have two $3 \pi / 4$ angles and three right angles. However, then two consecutive right angles are inevitable, contradicting Lemma 7. Notice that the hypothesis of the lemma is fulfilled: right angles are not mixed and hence no inactive sides.

Quadrilaterals. There are two cases. First consider rectangular chunks. At least one of its sides must be active, otherwise $u=0$. Since right angles are not mixed, all sides must be active. However, then, from Lemma 4 and the adjacency relation in $A_{u}$, the chunk must be one of the squares in Fig. 1. Second, the chunk may have two $3 \pi / 4$ angles and two $\pi / 4$ angles and must be the trapezoidal tile in Fig. 1.

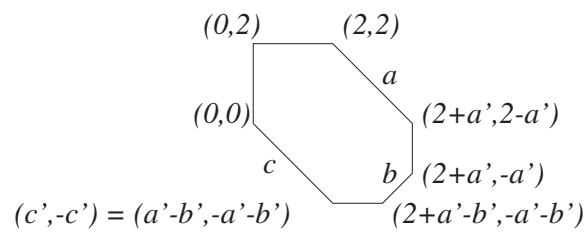

Fig. 13. There are no heptagonal chunks: $a=\sqrt{2} a^{\prime}, b=\sqrt{2} b^{\prime}$ and $c=\sqrt{2} c^{\prime}$. 
Triangles. The triangle must be right isosceles (Fig. 1).

Trapezoidal and triangular chunks have dashed sides: $\pi / 4$ angles are mixed. The upshot of the classification is the following-the possible chunks in a splitting $\Phi_{b}(u)$ are the tiles in Fig. 1: $\Phi_{b}(u)$ is a tiling. Thus, the function $\Phi_{b}$ indeed takes nonzero vectors in the kernel of $B W$ to the subset $\mathcal{T}_{b}$ of tilings. There is an analogous function $\Phi_{w}$ from ker $W B \backslash\{0\}$ to $\mathcal{T}_{w}$.

\section{5. $\Phi_{b}$ is a Bijection}

We start by showing that $\Phi_{b}$ : $\operatorname{ker} B W \backslash\{0\} \rightarrow \mathcal{T}_{b}$ is injective. Take $u, v \in \operatorname{ker} B W$, inducing tilings $\Phi_{b}(u)$ and $\Phi_{b}(v)$. We have to show that if $\Phi_{b}(u)=\Phi_{b}(v)$, then $A_{u}=A_{v}$ (and then, trivially, $u=v$ ). If $\Phi_{b}(u)=\Phi_{b}(v)$, then $A_{u}$ and $A_{v}$ may only differ on $\partial R$, since the only sides which may be active or not lie there. It is the shape of the tile which determines which side is active or not: inactive sides are the large basis of trapezoidal tiles and short sides of right triangles. Thus, sides of a tile in $\Phi_{b}(u)=\Phi_{b}(v)$ belonging to $\partial R$ must be simultaneously active or not in both $u$ and $v$, and the proof of injectivity is complete.

We now consider the surjectivity of $\Phi_{b}$. Given a tiling $T \in \mathcal{T}_{b}$ (and in particular, the description of type of activity for each side), we have to find a nonzero vector $u \in \operatorname{ker} B W$ for which $\Phi_{b}(u)=T$. The sequence of steps below is natural.

- Defining $u$ from $T$.

$u(b)=1 \Leftrightarrow b$ belongs to a nondashed side in $T$.

- Checking that $u \in \operatorname{ker} B W$.

We must show that, for each white point $w$, the sum of the values $u(b)$ for its neighbors $b \in G$ is even. By checking for each kind of tile, it is clear that the sum of $u(b)$ for neighbors $b$ of $w$ in a single tile is even. Thus, if $w$ lies in the interior of a tile, we are done. Suppose now that $w$ lies in a side of a tile of $T$. First, notice that vertices of tiles belonging to active sides are black. Hence, if $w$ is such a vertex, it must belong to inactive sides, and thus, both sides containing $w$ lie in $\partial R-w$ must be a corner of $R$, belonging to a right triangle. In this case the black neighbors of $w$ are active, if the short sides of the triangle are of length 1 , or inactive, for longer lengths. In both cases the total sum of $u(b)$ over black neighbors $b$ of $w$ is even.

Finally, if $w$ belongs to a side of a tile without being a vertex, this side must be either horizontal or vertical, since diagonal sides only cross black points. If this side lies in $\partial R$, all the black neighbors of $w$ belong to the same tile, and we are done. If, instead, this side is in the interior of $R$, we may take it to be horizontal and it must be an active side. Thus, $u\left(b_{1}\right)=u\left(b_{2}\right)=1$, where $b_{1}$ and $b_{2}$ are the side neighbors of $w$. Besides, the black neighbors of $w$ belong to two tiles, since $w$ is not a vertex. Suppose $b_{1}, b_{2}$ and $b_{3}$ are in one tile and $b_{1}, b_{2}$ and $b_{4}$ are in another. Then $u\left(b_{1}\right)+u\left(b_{2}\right)+u\left(b_{3}\right)$ and $u\left(b_{1}\right)+$ $u\left(b_{2}\right)+u\left(b_{4}\right)$ are even. Since $u\left(b_{1}\right)=u\left(b_{2}\right)=1$, we must have $u\left(b_{3}\right)=u\left(b_{4}\right)=0$. Hence, $u\left(b_{1}\right)+u\left(b_{2}\right)+u\left(b_{3}\right)+u\left(b_{4}\right)=0(\bmod 2)$. 


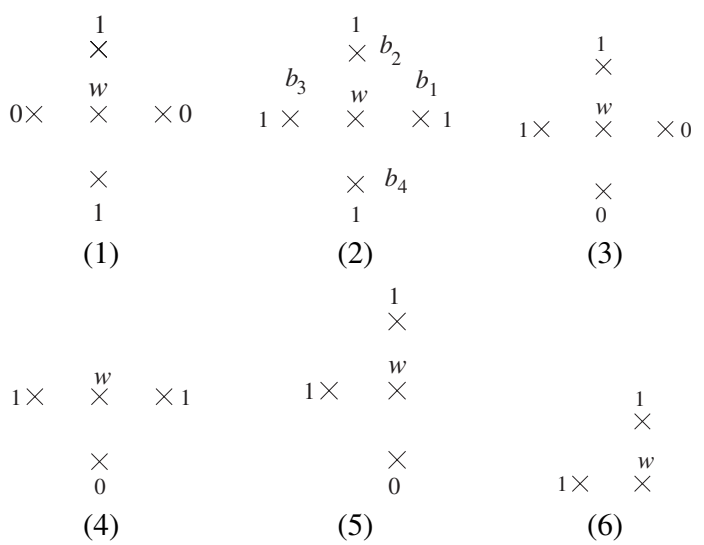

Fig. 14. Nontrivial active neighborhoods.

- The vector $u$ obtained from $T$ indeed satisfies $\Phi_{b}(u)=T$.

The black points by which nondashed sides of $T$ and $\Phi_{b}(u)$ passes are the same. Indeed, these are the active points of $u$. We are left with checking that the segments between these points are the same in $T$ and $\Phi_{b}(u)$. Clearly, $\partial R$ consists of segments common to both tilings. Therefore dashed sides are irrelevant: we only need to prove that $T$ and $\Phi_{b}(u)$ have the same active segments.

The active neighborhood of a point $w \in G$ under $u \in \operatorname{ker} B W$ is the set of its active black neighbors in $G$. An active neighborhood is trivial if there are no active points. A simple check obtains all nontrivial active neighborhoods: they are listed in Fig. 14, up to rotations. In cases (1), (2) and (3), $w$ is an interior point; in (4) and (5), $w$ belongs to a side of $\partial R$, and in (6), $w$ is a corner of $R$. Trivial active neighborhoods are not relevant in the forthcoming argument. In order to show that $T$ and $T_{\Phi}$ have the same active segments, all we have to do is to show this fact for each active neighborhood.

In the case of a neighborhood of type (1), the two active points are joined by a segment in $\Phi_{b}(u)$, by definition. Suppose that this segment does not belong to $T: w$ then is in the interior of a tile $t$ of $T$. The two active neighbors of $w$ belong to the boundary of $t$, by the construction of $u$ from $T$. Since tiles are convex, the segments of $T$ form angles which are less than or equal to $\pi$. Also, sides of tiles in $T$ do not pass by points at which $u$ is zero (we do not have to take into account boundary points). Thus, the sides of $t$ passing by the active points of the neighborhood of $w$ must be horizontal, as shown in Fig. 15; these sides extend to active neighbors at distance 2. However, by Fig. 1 horizontal sides have length at most 2 - contradiction. Thus $T$ equals $\Phi_{b}(u)$, when restricted to an active neighborhood of type (1).

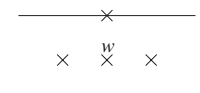

Fig. 15. The boundary of tile $t$. 


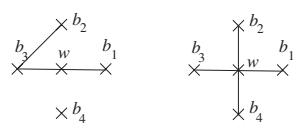

(1)

(2)

Fig. 16. Active segments

A
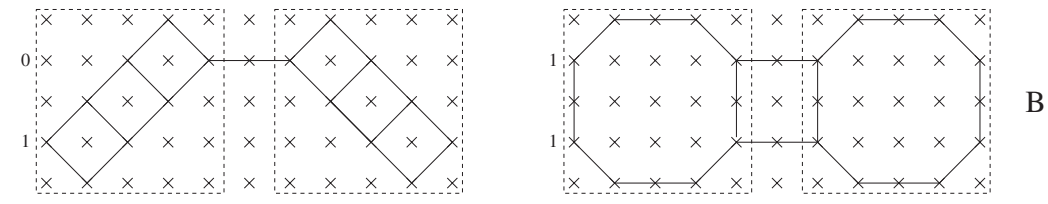

$\mathrm{A}+\mathrm{B}$
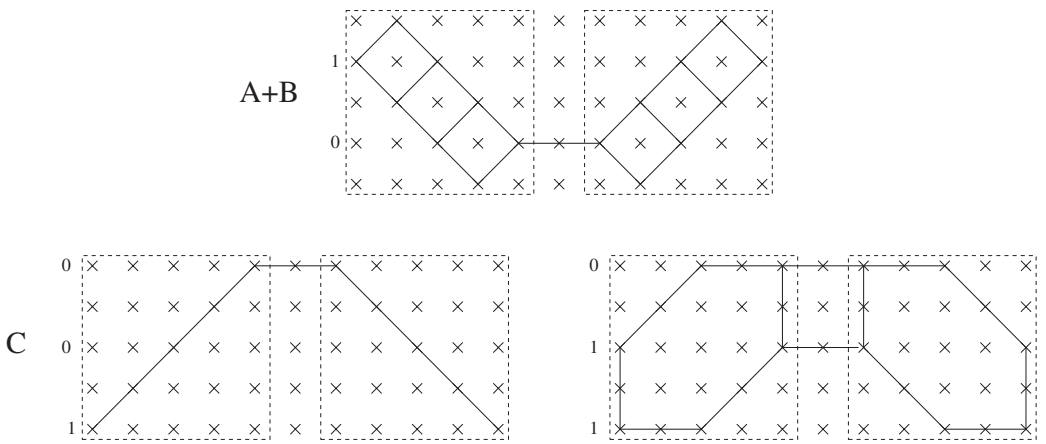

E

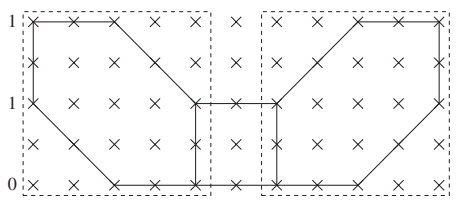

$\mathrm{C}+\mathrm{D}$
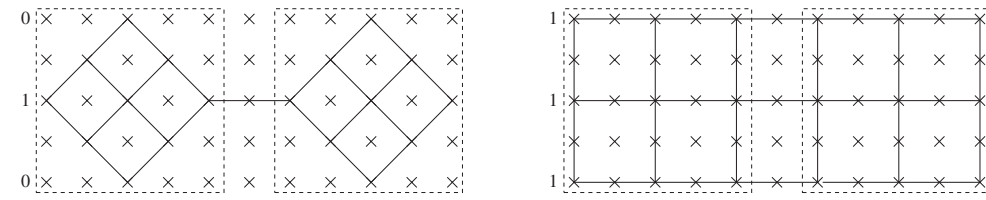

$\mathrm{C}+\mathrm{E}$
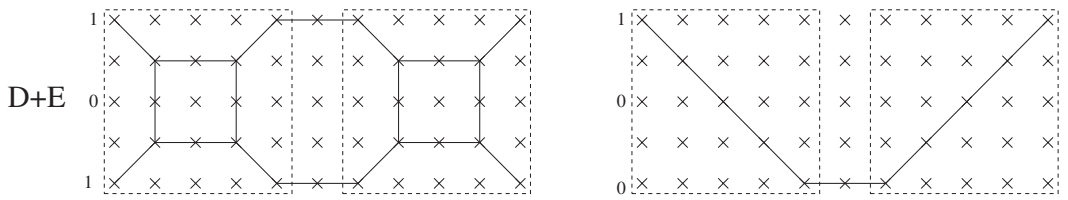

Fig. 17. All tilings of the $10 \times 4$ rectangle. 
For a neighborhood of type (2), $T$ contains active segments joining each neighbor of $w$ to some other neighbor. Consider the possibility of one of these segments being horizontal, as $\overline{b_{1} b_{3}}$ in Fig. 16(1). Neighbor $b_{2}$ is either joined to $b_{3}$ or to $b_{4}$, up to trivial symmetries, as in Fig. 16. Tiling $T$ cannot contain (1), since the $\pi / 4$ angle may only appear at $\partial R$ (again, check the shape of tiles in Fig. 1). Possibility (2) may not happen either, since it implies a white vertex of a tile which is not a corner of $R$. Thus segment $\overline{b_{1} b_{3}}$ is not in $T$. Vertical segments are equally excluded.

Suppose now that there are only diagonal active segments. The only tile with parallel diagonal sides $\sqrt{2}$ apart is the tilted square. Thus, the four diagonal segments must appear in both $T$ and $\Phi_{b}(u)$.

The study of neighborhoods of types (3)-(6) is similar and will be left to the reader. This proves the surjectivity of $\Phi_{b}$.

At this point, the proof of Theorem 2 is complete.

A consequence of the theorem is the fact that tilings of the same color can be added, since they correspond to kernel elements of the same matrix. Figure 17 shows all tilings of the $10 \times 4$ rectangle: the nonzero elements in the kernel of $W B$ give rise to the first three, $A, B$ and $A+B$. The remaining ones correspond to nonzero elements in the kernel of $B W$.

We now consider the lifting of polarized $\mathbb{Z} /(2)$-harmonic functions. Again, a tiling $T$ corresponds to a vector $u \in \operatorname{ker} B W_{\mathbb{Z} /(2)} \cup \operatorname{ker} W B_{\mathbb{Z} /(2)}$. Consider its graph $A_{u}$ of active points: from the shape of the tiles, each loop in the graph has even length. In particular, active points may be labeled 1 and -1 alternatively: this defines the lifting of $u$ to a vector with coordinates equal to 0,1 and -1 which may be interpreted as lying in $\operatorname{ker} B W_{K} \cup \operatorname{ker} W B_{K}$. The connectivity of $A_{u}$ is all we need to prove the result below.

Theorem 4. Lifting is a bijection between polarized $\mathbb{Z} /(2)$-harmonic functions and classes of vectors, $[v,-v]$, where $v \in \operatorname{ker} B W_{K} \cup \operatorname{ker} W B_{K}$ with coordinates equal to 0 , 1 and -1 .

\section{Acknowledgment}

The authors are thankful to Nicolau Saldanha for many conversations.

\section{References}

[C] Collatz, L., The Numerical Treatment of Differential Equations, Springer-Verlag, Berlin (1966).

[DT] Deift, P. A., and Tomei, C., On the determinant of the adjacency matrix for a planar sublattice, J. Combin. Theory Ser. B 35(3), 278-289 (1983).

[EKLP] Elkies, N., Kuperpberg, G., Larsen, M., and Propp, J., Alternating sign matrices and domino tilings, J. Algebra Combin. 1, 112-132 and 219-239 (1992).

[K] Kasteleyn, P. W., The statistics of dimers on a lattice, I. The number of dimer arrangements on a quadratic lattice, Physica 27, 1209-1225 (1961).

[ST1] Saldanha, N., and Tomei, C., Tilings of quadriculated annuli. URL: http://www.arxiv.org/math. $\mathrm{CO} / 0012265$.

[ST2] Saldanha, N., and Tomei, C., Arithmetic properties of the adjacency matrix of quadriculated disks. URL: http://www.arxiv.org/math.CO/0107162.

[STCR] Saldanha, N., Tomei, C., Casarin, M., and Romualdo, D., Spaces of domino tilings, Discrete Comput. Geom. 14, 207-233 (1995).

Received August 8, 2001, and in revised form January 22, 2002. Online publication July 10, 2002. 